OPEN ACCESS

Edited by:

Sujogya Kumar Panda, KU Leuven, Belgium

Reviewed by:

Melyssa Negri,

State University of Maringá, Brazil

Renátó Kovács,

University of Debrecen, Hungary

*Correspondence:

Yue Qu

yue.qu@monash.edu

tThese authors have contributed equally to this work

Specialty section: This article was submitted to Antimicrobials, Resistance and Chemotherapy,

a section of the journal Frontiers in Microbiology

Received: 20 August 2019 Accepted: 25 October 2019 Published: 07 November 2019

Citation:

Wu X, Zhang S, Xu X, Shen L, Xu B, Qu W, Zhuang W, Locock K,

Deighton M and Qu Y (2019) RAFT-Derived Polymethacrylates as a Superior Treatment for Recurrent Vulvovaginal Candidiasis by Targeting Biotic Biofilms and Persister Cells. Front. Microbiol. 10:2592. doi: 10.3389/fmicb.2019.02592

\section{RAFT-Derived Polymethacrylates as a Superior Treatment for Recurrent Vulvovaginal Candidiasis by Targeting Biotic Biofilms and Persister Cells}

\author{
Xueqing Wu ${ }^{1,2 t}$, Sisi Zhang ${ }^{2 t}$, Xinxin $X u^{2}$, Laien Shen ${ }^{2}$, Boyun $X u^{2}$, Wenzhen $Q u^{2}$, \\ Wenyi Zhuang', Katherine Locock ${ }^{3,4}$, Margaret Deighton ${ }^{5}$ and Yue $Q u^{6,7 *}$ \\ ${ }^{1}$ The Division of Gynecology, Shenzhen University General Hospital, Shenzhen, China, ${ }^{2}$ Department of Obstetrics \\ and Gynecology, Wenzhou Medical University, Wenzhou, China, ${ }^{3}$ CSIRO Manufacturing Flagship, Clayton, VIC, Australia, \\ ${ }^{4}$ School of Chemical and Biomedical Engineering, The University of Melbourne, Melbourne, VIC, Australia, ${ }^{5}$ School \\ of Applied Sciences, RMIT University, Bundoora, VIC, Australia, ${ }^{6}$ Neonatal Intensive Care Unit, The Second Affiliated \\ Hospital and Yuying Children's Hospital of Wenzhou Medical University, Wenzhou, China, ${ }^{7}$ Department of Microbiology, \\ Monash University, Clayton, VIC, Australia
}

Background: Vulvovaginal candidiasis (VC) is a common infection in need of more effective treatment. Formation of epithelium-associated Candida biofilms and the presence of persister cells are among the major contributing factors to the recurrence of this condition. We have previously developed RAFT-derived polymethacrylates that are effective in killing $C$. albicans biofilms in vitro. This study aimed to examine the clinical potential of polymethacrylates as antifungals for treatment of recurrent WVC (RVVC).

Methods: A mouse model of WV was used to establish vaginal epithelium-associated biofilms, using C. albicans isolates from WVC/RVVC patients. A comparison was made of the efficacies of polymethacrylates and conventional antifungals, clotrimazole and nystatin, in killing Candida in epithelium-associated biofilms in vivo. Ex vivo biofilms were used for Candida population profiling and to quantify persister cells in vaginal epithelia. The potency of polymethacrylates and conventional antifungals against persister cells, either as sole agents or in combination, was assessed.

Results: Polymethacrylates showed negligible local toxicity, resistance to vaginal acidity, and outstanding in vivo activity against vaginal epithelium-associated C. albicans biofilms. In vivo tests polymethacrylates outperformed the conventional antifungals, nystatin and clotrimazole at concentrations 50 times below the over-the-counter concentrations. Using polymethacrylates was associated with fewer persister cells, and better eradication of persister cells pre-selected by conventional antifungals.

Conclusion: This study systematically assessed the clinical potential of RAFTderived polymethacrylates as an effective treatment for WC/RWC in a mouse model. Polymethacrylates effectively killed vaginal epithelium-related C. albicans in vivo by specially targeting biotic biofilms and persister cells. Treatment presented negligible local toxicity.

Keywords: RVVC, mouse model, antifungal treatment, RAFT-derived polymethacrylates, biotic biofilms, persister cells, population analysis 


\section{INTRODUCTION}

Vulvovaginal candidiasis (VVC) is one of the most common infections in women, affecting up to $75 \%$ of those of reproductive age. A 5-8\% subset of these women suffer from the very stubborn recurrent VVC (RVVC, at least 3-4 episodes within a 12month period), which is resistant to currently available treatment (Sobel, 2016). Topical preparations, such as clotrimazole cream or nystatin suppositories are used as first-line antifungals for VVC and RVVC (Wächtler et al., 2011; Choukri et al., 2014). Fluconazole is also recommended by CDC as an effective oral antifungal for induction and maintenance therapy of RVVC. Although most C. albicans isolates from RVVC patients remain sensitive to many azoles (Gamarra et al., 2014; Adjapong et al., 2017), suppressive maintenance therapies using azoles often fail to eradicate the pathogen or completely cure the infection (Fan et al., 2015a; Mendling et al., 2015; Grinceviciene et al., 2017), suggesting microbial strategies other than intrinsic resistance might be involved.

Biofilm formation by C. albicans on vaginal epithelia has been long recognized as a specific fungal self-protective strategy that leads to antifungal resistance and infection recurrence (Harriott et al., 2010; Muzny and Schwebke, 2015), although its physical presence in the vagina of VVC/RVVC patients was recently questioned (Sobel, 2016; Swidsinski et al., 2019). Persister cells are a small population of "transiently resistant" cells that are often associated with the specific mode of biofilm growth (Lafleur et al., 2006; Lafleur et al., 2010; Yang et al., 2015). It is reasonable to hypothesize that epithelium-associated C. albicans biofilms involved in RVVC may harbor persister cells. Treatment of persister cells is known to be troublesome (Lewis, 2010). Conventional antimicrobials, unless used at the very high doses for an extended period, often fail to eradicate persister cells residing in biofilms (Yang et al., 2015).

Fluconazole maintenance suppressive therapy is still a preferred option to treat RVVC caused by fluconazole-sensitive C. albicans (Mikamo et al., 2015; Dennerstein, 2017). Other conventional antifungals that have a broad spectrum of activity, such as imidazole and nystatin, have been recommended for RVVC caused by fluconazole-resistant C. albicans and other Candida species (Fan et al., 2015a,b). Several limitations of using conventional agents to treat RVVC have been reported, including low efficacy in curing RVVC, possible systemic sideeffects, and the development of antifungal resistance (Howley et al., 2016; Molgaard-Nielsen et al., 2016). The biofilm growth model of Candida species around vaginal epithelia, the possible involvement of persister cells, and the acidic condition of the human vagina, have been shown to reduce the effectiveness of some conventional and newly developed antifungals against Candida spp. (Kasper et al., 2015; Muzny and Schwebke, 2015). These factors highlight the importance of developing new, effective and safe antifungal drugs that are able to overcome such hurdles for the treatment of VVC/RVVC.

We have previously developed biocompatible RAFT-derived cationic polymethacrylates and demonstrated their efficacy against in vitro biofilms formed by C. albicans (Qu et al., 2016). The aim of this study was to systematically evaluate the potential of the polymethacrylates for the treatment of VVC/RVVC, by examining their local toxicity, in vivo efficacy in killing biotic biofilms and persister cells, and tolerance to acidic conditions encountered in the human vagina, using a mouse model.

\section{MATERIALS AND METHODS}

\section{C. albicans Strains, Antifungal Agents and Ethical Approval}

Two C. albicans clinical isolates and DAY185, a well-known biofilm-producing laboratory strain, were used in this study. The clinical isolates were from patients who visited the first Affiliated Hospital, Wenzhou Medical University with clinically diagnosed uncomplicated VVC and RVVC and were designated as isolates VVC2 and VVC4, respectively. The clinical isolates were identified to a species level using CHROMagar Candida medium (CHROMagar, Paris, France) and MALDI Biotyper Identification System (MALDI-TOF MS, BioMérieux, Craponne, France). All strains were stored at $-80^{\circ} \mathrm{C}$ in $15 \%(\mathrm{v} / \mathrm{v})$ glycerol and streaked onto yeast-peptone-dextrose plates (YPD, $2 \%$ peptone, $1 \%$ yeast extract, $2 \%$ glucose and $80 \mathrm{mg} / \mathrm{L}$ uridine) as working stocks. The conventional antifungals for laboratory use, nystatin, clotrimazole, and amphotericin B, were purchased from Merck Pty Ltd. (Nantong, China). The clinically used antifungal preparations, nystatin vaginal suppository and clotrimazole cream were purchased from Polichem S.R.L (Barcelona, Spain) and Bayer Schering Parma AG (Berlin, Germany), respectively. Two polymethacrylates, designated as KL706 and KL708 and with similar compositions to those described previously, were used in this study (Table 1; $\mathrm{Qu}$ et al., 2016). The Ethics Review Boards of Wenzhou Medical University approved this study (wydw2016-0214). All animal experiments were carried out in accordance with the National Institutes of Health guide for the care and use of Laboratory animals.

\section{Formulation of Antimicrobial Polymethacrylate Hydrogels}

Both polymethacrylates were formulated to final concentrations of 20,40 , and $80 \mathrm{mg} / \mathrm{L}$, respectively in hydrogel preparations using a proprietary Carbopol $^{\circledR} 940$ polymer as follows: Synperonic ${ }^{\mathrm{TM}} \mathrm{PE} / \mathrm{F} 127$ emulsifier $(10 \mathrm{~g})$ was dissolved in water $(429 \mathrm{~mL})$ with heating and then cooled to room temperature before the addition of a stock solution of polymethacrylate $(0.01-0.04 \mathrm{~g})$ in ethanol $(5 \mathrm{~mL})$ and wetted out completely. To do this, the solution was stirred vigorously before the addition of propylene glycol $(50.0 \mathrm{~g})$, followed by the slow addition of Carbopol $^{\circledR} 940$ polymer $(5.00 \mathrm{~g})$. The final mixture was brought to $\mathrm{pH} 7.0$ using triethanolamine $(0.75 \mathrm{~g})$.

\section{Qualitative Determination of the Intravaginal Dynamics of Polymethacrylates}

Release of polymethacrylates from the formulated hydrogel in the mouse vagina over a time course was determined, 
TABLE 1 | Details of antimicrobial polymethacrylates used in this study, KL-706 and KL-708 (Locock et al., 2014).

Polymer Structure

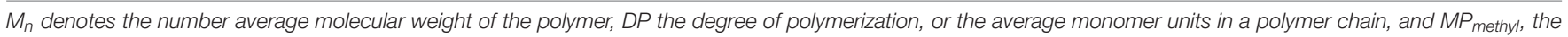
mole percentage of methyl side chains in the molecule, in this case compared with amino side chains.

using a previously published method (Zhang et al., 2017). Hydrogel loaded with rhodamine-labeled KL708 at $80 \mathrm{mg} / \mathrm{L}$ was administered to mice via an intravaginal pathway. Animals were sacrificed at $1,2,8,12,24$, and $48 \mathrm{~h}$ and the entire vagina was collected and washed with saline. Fluorescent images of the vagina were taken using an IVIS spectrum in vivo imaging system at $550 \mathrm{~nm}$.

\section{In vivo Cervicovaginal Toxicity and Inflammation Assessment}

A mouse model was used to assess cervicovaginal toxicity and local inflammation after polymethacrylate exposure (Catalone et al., 2004). BABL/C female mice of 6-8 weeks old were hormonally synchronized 3 days prior to the experiment with a subcutaneous injection of $0.1 \mathrm{mg}$ of estrogen (17 $\beta$-estradiol; Sigma) dissolved in $0.1 \mathrm{~mL}$ sesame oil. Anesthetized mice received an intravaginal treatment $(100 \mu \mathrm{L})$ of either unformulated hydrogels (negative control) or KL708-hydrogel. PBS was used as the second negative control to document the normal tissue architecture and inflammation status in the cervicovaginal mucosa. Mouse vaginal lavage fluids were collected and the vaginal tissue was surgically excised after sacrificing the animals at $48 \mathrm{~h}$ post-treatment. Tissues were formalin-fixed and embedded in paraffin using standard procedures. Gross morphological analyses of the cervicovaginal mucosa were performed on tissues stained with hematoxylin and eosin (H\&E) using an Olympus IX81 microscope. Concentrations of two representative inflammatory effectors in vaginal lavage fluids, the innate cytokine IL-1 $\beta$ (Netea et al., 2010) and the alarmin S100A8 (Vogl et al., 2014), were analyzed using commercially available enzyme-linked immunosorbent assay (ELISA) kits per manufacturers' instructions. All samples were measured in duplicate, and the assay was repeated three times.

\section{In vivo VVC Model and Cultivation of Mouse Vaginal Epithelium-Associated Candida Biofilms}

Vulvovaginal candidiasis was induced in mice essentially as described by Harriott et al. (2010). C. albicans cells were grown for $20 \mathrm{~h}$ in YPD $\left(200 \mathrm{rpm}, 30^{\circ} \mathrm{C}\right)$ and resuspended to a density of $\sim 7 \times 10^{6} \mathrm{CFU} / \mathrm{mL}$. One-hundred microliters of Candida suspension was used to infect mice via the intravaginal pathway. Infected vaginal tissue was surgically collected after euthanizing animals 3 days post-infection. C. albicans biofilms that formed on vaginal epithelia were qualitatively examined using scanning electronic microscopy (SEM).

\section{Antifungal Susceptibility Tests for Planktonic Cells and Cells Residing in Epithelium-Associated Biofilms}

Broth dilution susceptibility testing was carried out for C. albicans cells grown as planktonic cultures, by following the CLSI guideline M27-A3, except RPMI-1640 at pH 4.0 and pH 7.2 were used respectively, as growth media. Polymethacrylate solutions at concentrations ranging from 1 to $128 \mathrm{mg} / \mathrm{L}$ were tested. For in vivo biofilm antifungal susceptibility testing, C. albicans epithelium-associated biofilms were established in the mouse vagina as described above. Nystatin vaginal suppository and clotrimazole cream at $4000 \mathrm{mg} / \mathrm{L}$ (OTC preparations), and hydrogels loaded with KL706 and KL708 respectively, at 20, 40, and $80 \mathrm{mg} / \mathrm{L}$ were delivered as a single dose to infected vagina via an intravaginal pathway. Treatment lasted for $24 \mathrm{~h}$ before infected animals were sacrificed, and the entire vagina was surgically excised. The vaginal tissues were weighted with a Mettler Toledo PB603-S milligram balance, washed three times with PBS, and homogenized with a tissue homogenizer. Viable counts were performed for tissue suspensions by plating an aliquot onto YPD plates followed by further incubation at $35^{\circ} \mathrm{C}$ for $48 \mathrm{~h}$. 
Colony-forming-unit (CFU) of Candida cells per gram of vaginal tissue (CFU/g) was calculated for quantitative assessment of fungal biofilm survivors after antifungal treatment. Mouse vagina treated with PBS and blank hydrogels served as negative controls. Six biological repeats were carried out for this experiment.

\section{Population Profiling of Infected Vaginal Tissues and ex vivo Quantification of Persister Cells}

Profiling the population of $C$. albicans cells within infected tissues was carried out using a previously published method, with modification (Yang et al., 2015). Approximately $0.3 \mathrm{~g}$ of infected vaginal tissues were homogenized with a tissue homogenizer in $1 \mathrm{~mL}$ of RPMI-1640. Suspensions of Candida cells were adjusted to a density of $\sim 1 \times 10^{8} \mathrm{CFU} / \mathrm{mL}$ with RPMI-1640 and a hemocytometer, and further confirmed with CFU-based viable counts. One-hundred microliters of fungal suspension was challenged with nystatin, clotrimazole, and KL706 prepared in RPMI-1640 at increasing concentrations ranging from planktonic MIC to $1024 \mathrm{mg} / \mathrm{L}$ for clotrimazole, $2048 \mathrm{mg} / \mathrm{L}$ for nystatin, and $256 \mathrm{mg} / \mathrm{L}$ for KL706. Our preliminary study found such high concentrations were effective against vaginal epithelium-associated C. albicans biofilms ex vivo. Viable counts were performed after exposing fungal suspensions to antifungal agents at various concentrations for $24 \mathrm{~h}$, and the highest concentrations for an extra $24 \mathrm{~h}$ (48 $\mathrm{h}$ in total) and $48 \mathrm{~h}$ (72 $\mathrm{h}$ in total). To avoid antifungal carryover, the antifungal treated suspensions were centrifuged at $3000 \mathrm{~g}$ for 5 min, washed with PBS, and resuspended to the same volume of YPD broth. Viable counts were performed by plating serially diluted aliquots onto YPD plates followed by incubation at $35^{\circ} \mathrm{C}$ for $72 \mathrm{~h}$ to maximize the recovery of persister cells. The lowest concentration of antifungals and the shortest treatment duration that led to the lowest number of fungal survivors were chosen as the antifungal regimens for persister cell quantification. The percentage of persister cells were calculated as follows: (fungal density after antifungal treatment)/(fungal density before antifungal treatment $) \times(100 \%)$.

\section{Potency of Polymethacrylates in Further Killing Persister Cells Pre-selected by Conventional Antifungals}

As persister cells residing in biofilms might be antimicrobialdependent (Yang et al., 2015), we further evaluated the effectiveness of polymethacrylates in killing persister cells that have been pre-selected by conventional antifungals. A population of $\sim 1 \times 10^{8} \mathrm{CFU} / \mathrm{mL}$ of fungal cells from infected vaginal tissues were treated with amphotericin $B$ at $100 \mathrm{mg} / \mathrm{L}$ for $24 \mathrm{~h}$, washed with PBS to remove the amphotericin B, and then subjected to treatment using nystatin $(2048 \mathrm{mg} / \mathrm{L})$, or clotrimazole $(1024 \mathrm{mg} / \mathrm{L})$, or KL706 (80 mg/L) or KL708 $(80 \mathrm{mg} / \mathrm{L})$ for another $24 \mathrm{~h}$. Viable counts were carried out to recover and quantify survivor persister cells as described earlier. To increase the detection sensitivity, a one $\mathrm{mL}$ volume of the treated suspension was pelleted, washed three times with PBS, and resuspended into $100 \mu \mathrm{L}$ of PBS, followed by spreading and recovering on YPD plates. The experiment was repeated three times in duplicate.

\section{Data Analysis and Statistical Methods}

One-way ANOVA or a non-parametric test (Mann-Whitney $U$ test) was carried out to compare two means, depending upon the data distribution. Statistical significance was assumed at the $p$-value of less than 0.05. Data analysis was performed using Minitab 16 (Minitab, State College PA, United States).

\section{RESULTS}

\section{Formulation of Polymer Loaded Hydrogels and Intravaginal Sustainability of RAFT-Derived Polymethacrylates}

Figure $\mathbf{1 A}$ is an example of a formulated hydrogel of polymethacrylate KL708. Figure 1B showed a gradual diminution of rhodamine-labeled KL708 from the hydrogel for a 48-h period after infusion into the mouse vagina. The slow decay of fluorescence intensity in the vagina supported the stability, for at least $48 \mathrm{~h}$, of such a drug delivery system in the vaginal environment and a prolonged contact between polymethacrylate and the vaginal epithelia, paving the foundation for intravaginal application of this system.

\section{Minimum Cervicovaginal Toxicity of RAFT-Derived Polymethacrylates}

Toxicity of polymethacrylates to cervicovaginal tissues was determined by examining the morphology of vaginal epithelia and local inflammation. Hematoxylin and eosin staining showed no significant change in the gross appearance of the vaginal mucosa after exposure to hydrogels loaded with KL708 at $40 \mathrm{mg} / \mathrm{L}$ or $80 \mathrm{mg} / \mathrm{L}$, or KL706 at $40 \mathrm{mg} / \mathrm{L}$ for $48 \mathrm{~h}$, when compared with hydrogel or non-hydrogel PBS controls (Figure 2A). No apparent epithelial disruption in the mouse vagina was noticed when challenged with these products. Vaginal epithelia remained protected by a covering of keratin, and the integrity of the squamous epithelia appeared intact (Figure 2A). Minor sloughing was observed when KL-706 was used at $80 \mathrm{mg} / \mathrm{L}$, however this involved only the upper epithelia (Figure 2A). No significant inflammatory responses were induced in mouse vagina by polymethacrylate-loaded hydrogels, even at a high polymer concentration of $80 \mathrm{mg} / \mathrm{L}$, as indicated by the level of inflammatory cytokine IL-1 $\beta$ and alarmin S100A8 (Figure 2B).

\section{Acidic Vaginal Environment Did Not Compromise Anti-candida Activity of Polymethacrylates}

Minimum inhibitory concentrations were determined for both conventional antifungals and polymethacrylates, using the standard broth-dilution method but with RPMI prepared at a neutral $\mathrm{pH}$ (7.2) and a $\mathrm{pH}$ comparable to that in the human vagina (4.0) respectively,. All but one of the MICs 

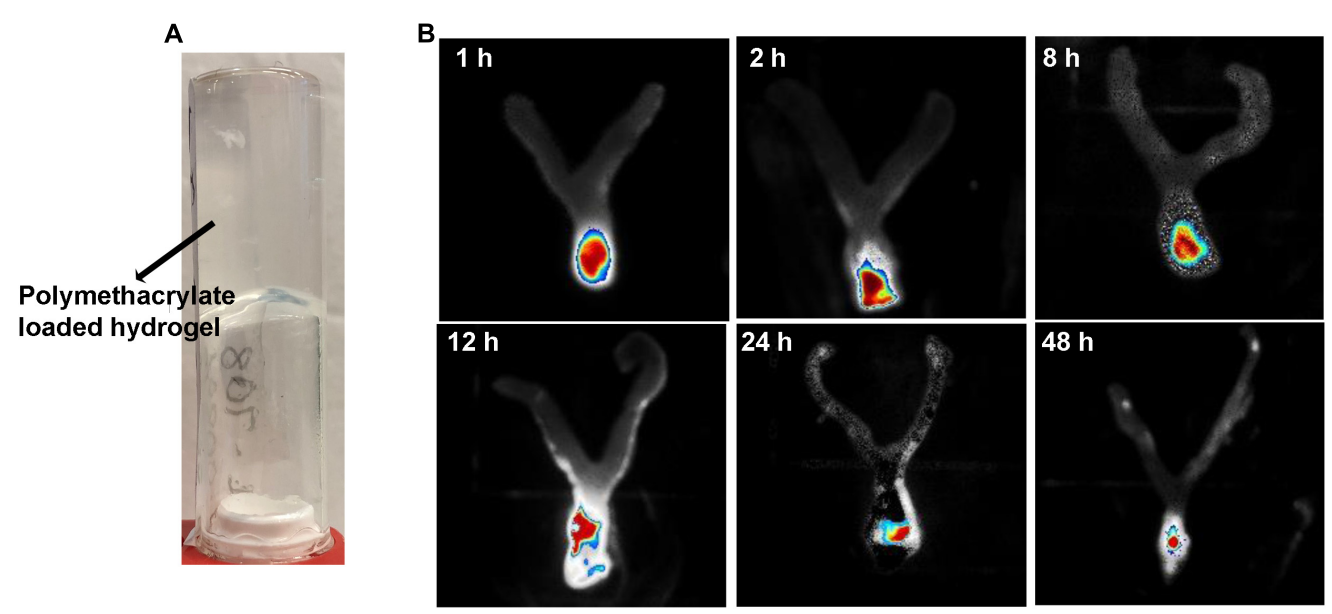

FIGURE 1 | Intravaginal retention and slow decay of polymethacrylates formulated as hydrogel in vivo. Fluorescence-labeled polymethacrylate KL-708 was formulated into hydrogels (A) and administered intravaginally to mice. Retention of guanylated polymer in mouse vagina was monitored in situ, using an in vivo IVIS spectrum imaging system at different time points of $0,2,8,12,24$, and $48 \mathrm{~h}(\mathbf{B})$. Substantial amounts of polymethacrylates were found in mouse vagina even at $48 \mathrm{~h}$ post-administration.

A
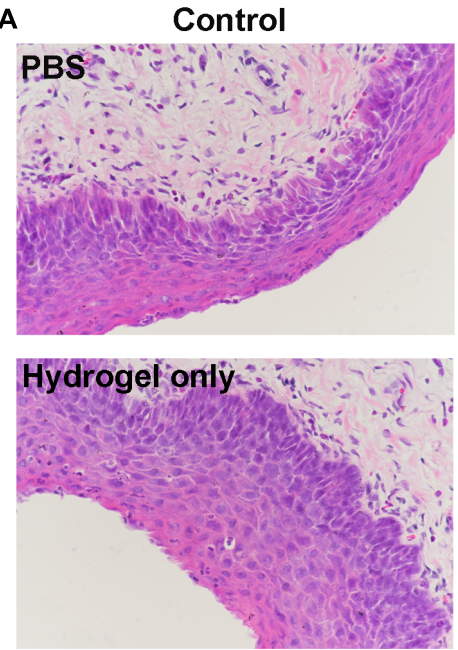

KL706 hydrogel

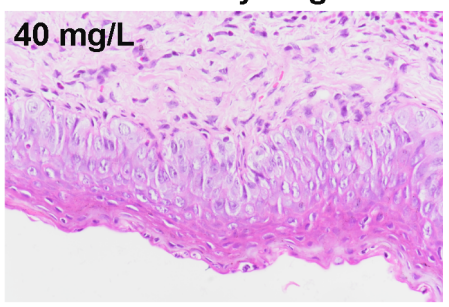

$80 \mathrm{mg} / \mathrm{L}$

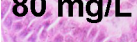

KL708 hydrogel

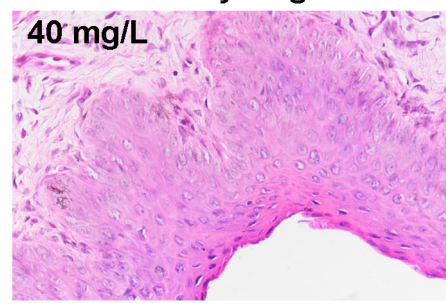

$80 \mathrm{mg} / \mathrm{L}$

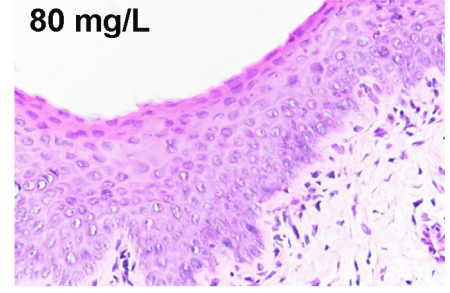

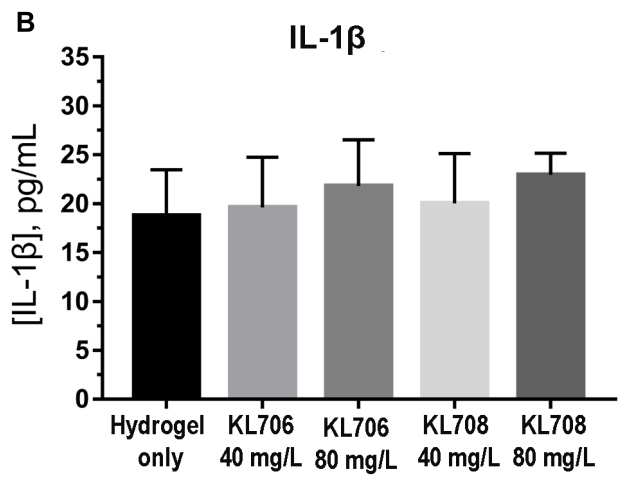

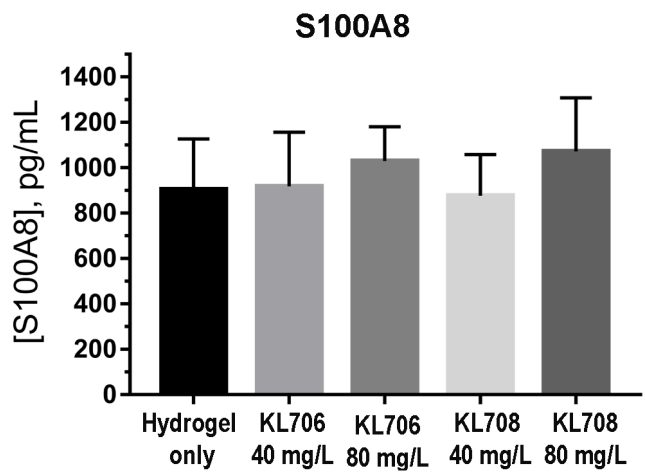

FIGURE 2 | Cervicovaginal toxicity of polymethacrylates and local inflammation. (A) Histopathological presentation of mouse vaginal tissues after exposure to polymethacrylate at 40 and $80 \mathrm{mg} / \mathrm{L}$ for $48 \mathrm{~h}$. HE staining showed neglectable morphological changes of vaginal epithelia and underlying tissues. (B) Inflammation responses induced by polymethacrylate-loaded hydrogels. Inflammatory cytokine IL-1 $\beta$ and alarmin S100A8 were used as indicators. 
derived for the polymethacrylates were identical under acidic conditions, indicating a high tolerance of these polymers to acidic conditions found within the human vagina (Table 2). The acidic condition, however, significantly compromised antifungal activities of conventional drugs, by increasing their MICs by 4-16-fold (Table 2).

\section{Polymethacrylates Were Superior to Conventional Antifungals in Killing Epithelium-Associated Biofilms in the Vagina}

We first examined in vivo biofilm formation of C. albicans on mouse vaginal epithelia at $48 \mathrm{~h}$ post-infection. A characteristic epithelium-associated biofilm structure was observed with SEM, consisting of yeast and hyphal cells embedded in vaginal mucosae (Figure 3A, with the clinical isolate VVC4 as an example). We further examined the antifungal efficacy of all agents against such epithelium-associated biofilms formed by VVC4 and laboratory strain DAY185. KL706 and KL708 at $40 \mathrm{mg} / \mathrm{L}$ were effective in killing epithelium-associated biofilms formed by either C. albicans DAY185 or VVC4 (Figures 3A,B). KL706 at $80 \mathrm{mg} / \mathrm{L}$, a concentration 50 times less compared to that of conventional antifungals tested, killed significantly more biofilms than either nystatin or clotrimazole at $4000 \mathrm{mg} / \mathrm{L}(p<0.05$ and $p<0.01$, respectively). KL708 at $80 \mathrm{mg} / \mathrm{L}$ also showed a higher potency than clotrimazole at $4000 \mathrm{mg} / \mathrm{L}$ in killing epithelium-associated $C$. albicans biofilms in the mouse vagina (Figure 3B, $p<0.05$ ).

\section{Polymethacrylate Treatment of Epithelium-Associated C. albicans Biofilms Resulted in Fewer Persister Cells Than Conventional Antifungals}

Population analysis profiling was performed to determine antifungal regimens (concentration and duration) that can be used to successfully isolate and quantify persister cells residing

TABLE 2 | In vitro susceptibility of $C$. albicans strains to conventional antifungals and polymethacrylates at different $\mathrm{pHs}$.

\begin{tabular}{llccccc}
\hline Antifungals & \multicolumn{5}{c}{ MIC (mg/L) for C. albicans isolates } \\
\cline { 2 - 5 } & DAY185 & $\begin{array}{c}\text { Fold } \\
\text { change }\end{array}$ & VVC2 & $\begin{array}{c}\text { Fold } \\
\text { change }\end{array}$ & VVC4 & $\begin{array}{c}\text { Fold } \\
\text { change }\end{array}$ \\
\hline
\end{tabular}

\section{$\mathrm{pH}=7.2$}

Nystatin

Clotrimazole $\quad 0.5$

KL706 $\quad 16$

KL708 $\quad 16$

$\mathrm{pH}=\mathbf{4 . 0}$

Nystatin

Clotrimazole

$\mathrm{KL} 706$

16
8
32
32

16
4
14
2
2
2

in epithelium-associated C. albicans biofilms. Typical threesubpopulation patterns were established when nystatin and KL706 were used as the selecting agents (Figure 4A): Over 99.9\% of biofilm cells were susceptible and responded to both agents at $16 \mathrm{mg} / \mathrm{L}$; a small population of cells demonstrated tolerance to nystatin and KL706 at higher concentrations $(16-1024 \mathrm{mg} / \mathrm{L}$ for nystatin, and $16128 \mathrm{mg} / \mathrm{L}$ for KL706) but could be killed if antifungal concentration and/or exposure time were further increased; a very small population of cells survived the most potent antifungal regimens, such as nystatin at $2048 \mathrm{mg} / \mathrm{L}$ and KL706 at $256 \mathrm{mg} / \mathrm{L}$ for 48 or $72 \mathrm{~h}$. In contrast; a gradually descending pattern was found when fungistatic clotrimazole was used to select biofilm persister cells (Figure 4A). Thus, the regimens chosen to isolate persister cells in epithelium-based biofilms grown in the mouse vagina were $2048 \mathrm{mg} / \mathrm{L} \times 48 \mathrm{~h}$ for nystatin, $256 \mathrm{mg} / \mathrm{L} \times 48 \mathrm{~h}$ for KL706 and KL708, and $1024 \mathrm{mg} / \mathrm{L} \times 72 \mathrm{~h}$ for clotrimazole.

In general, fewer persister cells were detected after exposing vaginal epithelium-associated biofilm cells to KL706 or KL708 than to clotrimazole or nystatin (Figure 4B and Supplementary Table S1). When epithelium-associated biofilms formed by C. albicans VVC4 and VVC2 were studied, average proportions of persister cell selected by KL706 and KL708 reached $0.08 \pm 0.03 \%$ (mean \pm SEM) and $0.10 \pm 0.01 \%$, and $0.17 \pm 0.02 \%$ and $0.21 \pm 0.02 \%$, respectively. Conventional antifungal drugs nystatin isolated $0.16 \pm 0.02 \%$ and $0.28 \pm 0.02 \%$ persister cells, and clotrimazole selected $0.43 \pm 0.02 \%$ and $0.51 \pm 0.02 \%$ from biofilms formed by VVC4 and VVC2 respectively (Figure 4B). The same trend was noticed when C. albicans laboratory reference strain DAY185 was studied. It was also noticed that vaginal epithelium-based biofilms formed by clinical isolates VVC4 and VVC2 harbored more persister cells than C. albicans DAY185, a clinically relevant isolate related to systemic infections, regardless of selecting antifungals used.

\section{Polymethacrylates Demonstrate Activity Against Persister Cells Following Conventional Antifungal Treatment}

Ex vivo biofilms were used to examine the effectiveness of polymethacrylates in killing persister cells pre-selected by conventional antifungal agent amphotericin $\mathrm{B}$. When challenging persister cells preselected with amphotericin B at $100 \mathrm{mg} / \mathrm{L}$, polymethacrylates at $128 \mathrm{mg} / \mathrm{L}$ showed a significantly higher fungicidal activity relative to the conventional agents, nystatin and clotrimazole at $1024 \mathrm{mg} / \mathrm{L}$ (Figure 5). KL706 further killed 75,94 , and $82 \%$ of pre-selected persister cells of DAY185, VVC2, and VVC4, and KL708 eradicated 64, 90, and 75\% of these persister cells. This opens the door for novel and more efficient combination therapies for RVVC, using conventional antifungals sequentially combined with polymethacrylates.

\section{DISCUSSION}

The current antifungal regimens for VVC/RVVC, recommended by CDC and based on 2015 Sexually Transmitted Diseases (STD) Treatment Guidelines, have been widely followed by 
A

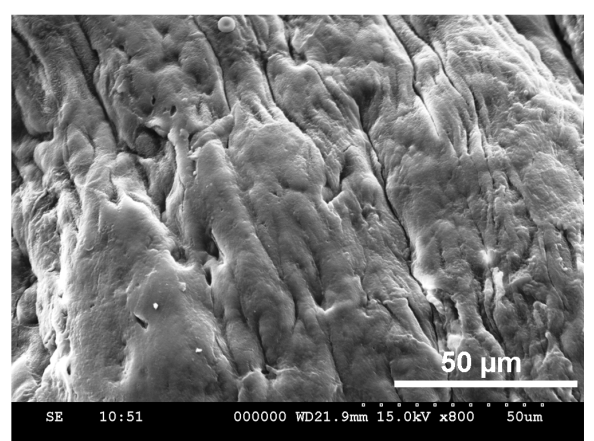

Control

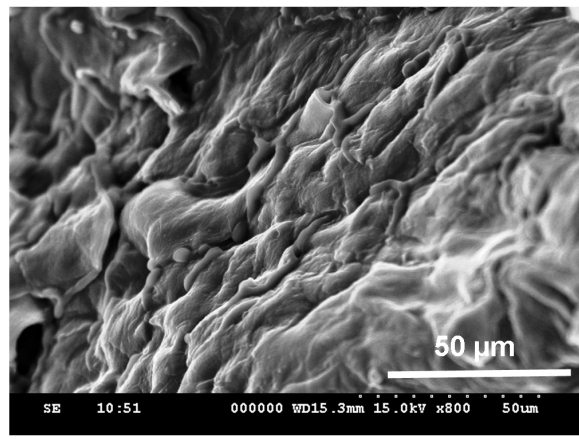

C. albicans VVC4 + KL708 (40 mg/L)

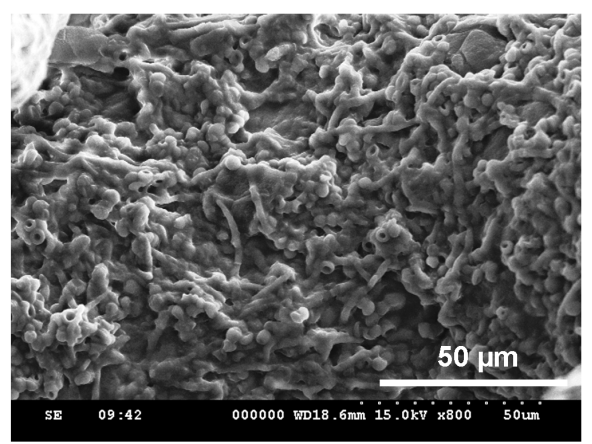

C. albicans VVC4

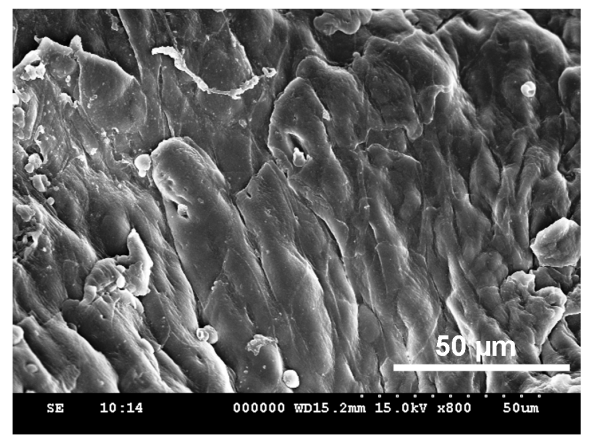

C. albicans VVC4 + KL708 (80 mg/L)

B

C. albicans DAY185

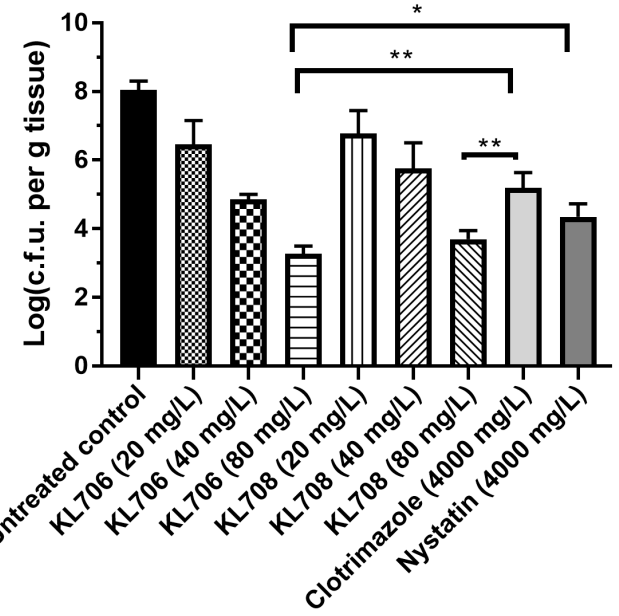

C. albicans VVC4

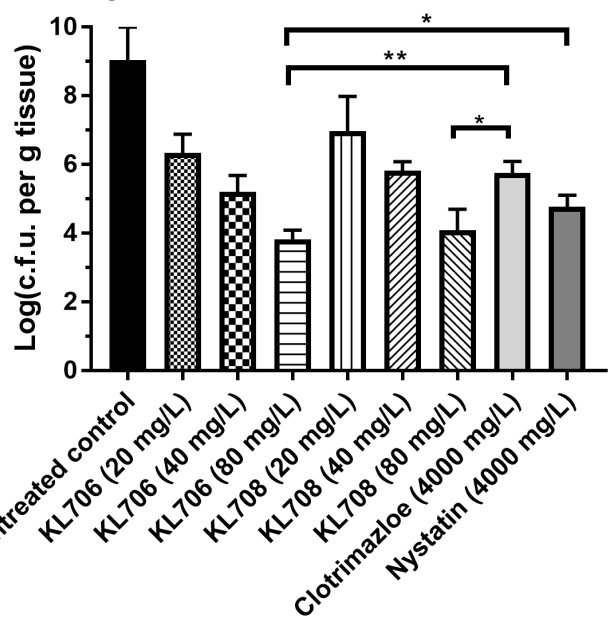

FIGURE 3 | Antifungal activities of polymethacrylates against C. albicans grown as vaginal epithelium-associated biofilms. (A) Scanning electron microscopy of epithelium-associated biofilms formed by C. albicans WVC4 in the mouse vagina, before and after treatment with KL708 at 40 and 80 mg/L. (B) Quantitative assessment of anti-infective efficacy of polymethacrylates against vaginal epithelium-associated C. albicans biofilms. C. albicans cells grown as biotic biofilms on mouse vaginal epithelia were treated with polymethacrylates at 40 and $80 \mathrm{mg} / \mathrm{L}$ and conventional antifungal drugs at clinical doses. Mouse vaginal tissue was removed after treatment and homogenized for viable counts. Survivors were recovered after $72 \mathrm{~h}$ incubation on YPD plates. Polymethacrylates at non-toxic concentrations $(80 \mu \mathrm{g} / \mathrm{mL})$ demonstrated a significantly higher activities in killing epithelium-associated biotic $C$. albicans biofilms than conventional antifungals. No difference was found between the two negative controls (PBS control and hydrogel-only control) and only that of hydrogel-only was shown. ${ }^{*} p<0.05,{ }^{* *} p<0.01$.

general practitioners and gynecologists. Among the conventional antifungals recommended by CDC, imidazoles (including clotrimazole, miconazole and econazole), the polyene nystatin, and fluconazole have been on the market for more than 35 years and still remain effective against acute VVC. Their effectiveness for RVVC, however, are suboptimal, as recurrence of infection is common after an initial course of fluconazole or clotrimazole and ongoing antifungal maintenance therapy is often required 

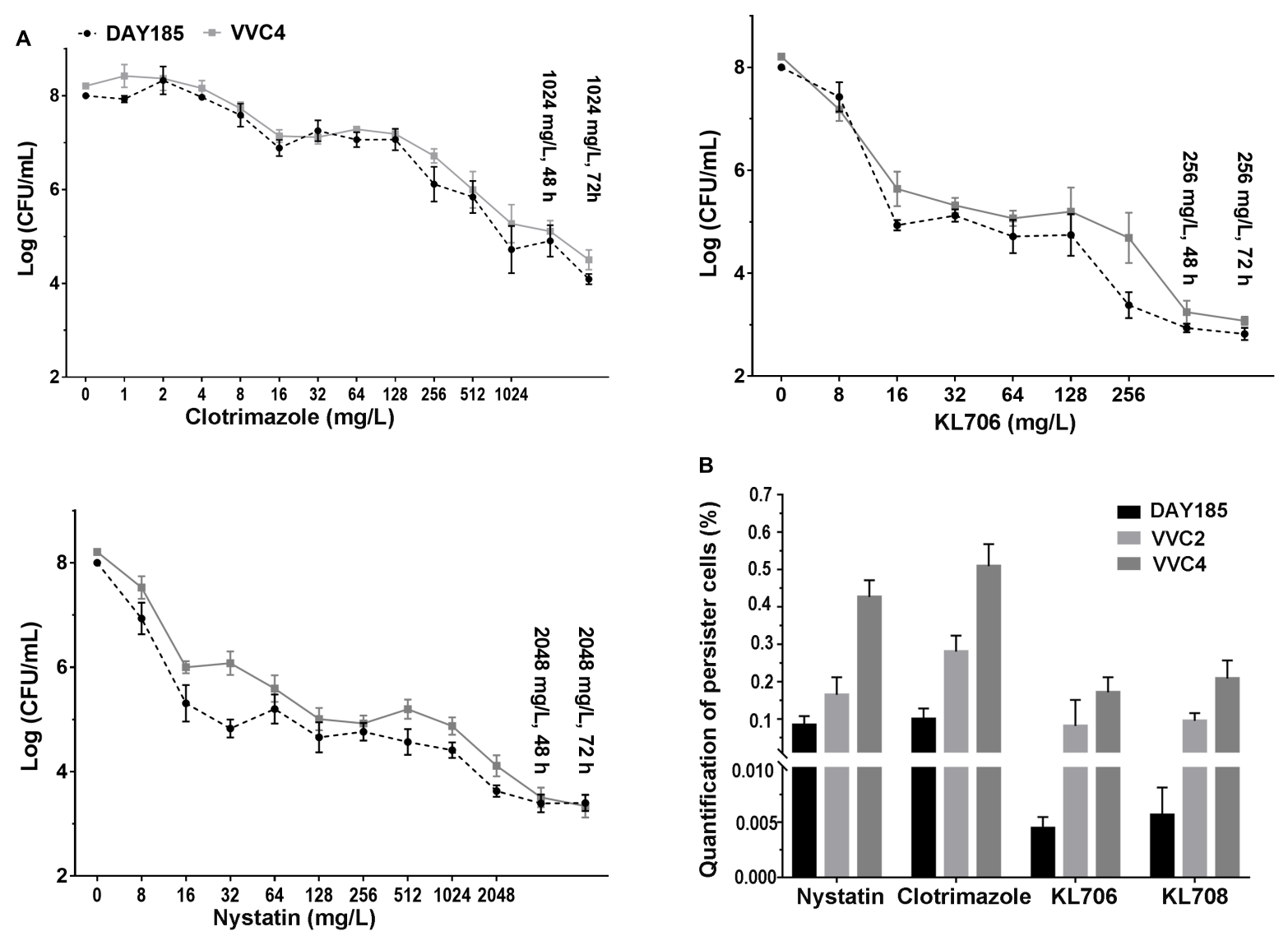

FIGURE 4 | (A) Ex vivo population profiling of vagina epithelium-associated C. albicans biofilms using different antifungal agents. Mice were infected with C. albicans via the intravaginal pathway. After 3 days, infected vaginal tissues were harvested and homogenized. Fungal suspensions containing persister cells were challenged with antifungals at increasing concentrations for 24,48 , and $72 \mathrm{~h}$. Experimental results identified a small population remaining tolerant to antifungals at very high concentration, even for an extended treatment period (48 or $72 \mathrm{~h}$ ). Polymers $\mathrm{KL} 706$ only required a relatively low concentration of $256 \mathrm{mg} / \mathrm{L}$ and $24-48 \mathrm{~h}$ to reach the persister cell plateau, however the conventional antifungal agents need a higher concentration of $1024 \mathrm{mg} / \mathrm{L}$ or $2048 \mathrm{mg} / \mathrm{L}$ to reach this plateau. (B) Proportions of $C$. albicans persister cells in epithelium-associated biofilms selected by different antifungal agents. Total persister cells were isolated by exposing homogenized mouse vaginal tissues infected with C. albicans to antifungal agents at a specific concentration $(1024 \mathrm{mg} / \mathrm{L}$ for clotrimazole, $2048 \mathrm{mg} / \mathrm{L}$ for nystatin, and $256 \mathrm{mg} / \mathrm{L}$ for KL706 and KL708, based on population analysis data). Such regimens have been found to be able to isolate persister cells from infected vaginal tissues. The proportion of persister cells was calculated as the proportion of survivor cells out of total population. Shown are the average of three to four biological repeats in duplicate.

(Tapia et al., 2017; Crouss et al., 2018). This is possibly due to the low efficacy of conventional antifungals in eradicating biotic biofilms and persister cells that have grown on vaginal epithelia and in underlying tissues.

We have recently developed a new class of antimicrobial polymethacrylates that have shown high potency against in vitro C. albicans biofilms (Qu et al., 2016). This study was designed to examine clinical potential of polymethacrylates as an effective treatment for VVC/RVVC. In the current study, polymethacrylates demonstrated several properties as topical agents for VVC/RVVC, including high efficacy in killing epithelia-based C. albicans biofilms and persister cells, ease of formulation as a hydrogel for topical application, and low toxicity to local vaginal tissue. Unlike other newly proposed antifungal substances from natural sources that often need to be combined with conventional agents such as fluconazole or miconazole for high in vivo effectiveness (Gao et al., 2016; Esposito et al.,
2018; Fernandes Costa et al., 2019), polymethacrylates provide a distinct advantage as they can be used as a sole therapy. They also outperformed clotrimazole and nystatin, two market leading antifungals, at 50 times less the equivalent clinical concentration in killing biotic $C$. albicans biofilms grown on vaginal epithelia. Moreover, polymethacrylates were able to maintain their activity in the acidic human vaginal environment, a major hurdles for drug development, since antifungals are often less effective under acid conditions (Ilkit and Guzel, 2011). Similar to valproic acid (Chaillot et al., 2017), both KL706 and KL708 demonstrated pHindependent antifungal efficacy, exhibiting only a 2-fold shift at $\mathrm{pH} 4.0$ compared with $\mathrm{pH} 7.2$, superior to many conventional antifungals and even the newly developed antifungal CD101 (Boikov et al., 2017).

The in vivo efficacy of polymethacrylates for VVC/RVVC found in this study is thought to be based on their unique activity against biotic biofilms and persister cells formed by C. albicans. 


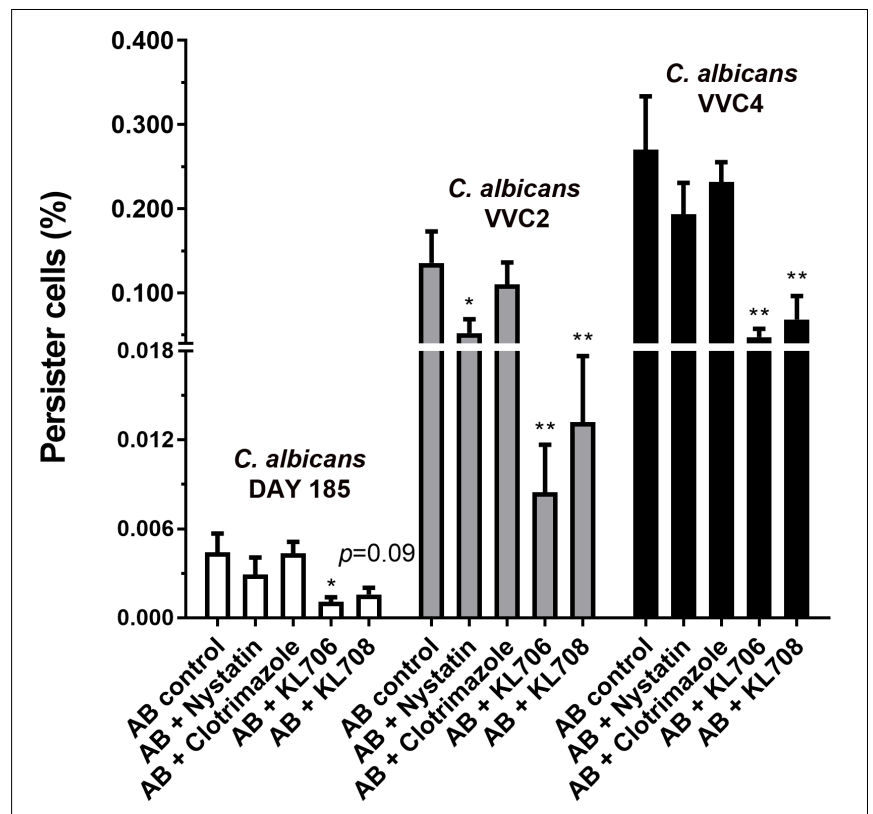

FIGURE 5 | Polymethacrylates demonstrate activity against persister cells pre-selected by conventional antifungals. Ex vivo biofilms were used to examine the effectiveness of polymethacrylates in killing persister cells pre-selected by conventional antifungal agent amphotericin B. Mice were infected with $C$. albicans via the intravaginal pathway. After 3 days, infected vaginal tissues were harvested and homogenized. Fungal suspensions containing persister cells were challenged with amphotericin B at $100 \mathrm{mg} / \mathrm{L}$ for $24 \mathrm{~h}$, followed by polymethacrylates (128 mg/L), nystatin (1024 mg/L) or clotrimazole (1024 mg/L) for another $24 \mathrm{~h}$. Viable counts were carried out to determine the number of survivor fungal cells. Polymethacrylates at $128 \mathrm{mg} / \mathrm{L}$ showed a significantly higher fungicidal activity relative to the conventional agents such as nystatin and clotrimazole at $1024 \mathrm{mg} / \mathrm{L}$. AB: amphotericin B; $A B+$ nystatin: fungal suspension was pre-treated with amphotericin $B$ $(100 \mathrm{mg} / \mathrm{L})$ for $24 \mathrm{~h}$ and then by nystatin at $1024 \mathrm{mg} / \mathrm{L}$ for another $24 \mathrm{~h}$. ${ }^{*} p<0.05,{ }^{* *} p<0.01$.

Antifungal resistance stemming from the formation of Candida biofilms in VVC has been noted by several studies, mostly based on antifungal susceptibility tests using 96-well microplate biofilm assays (Gao et al., 2016; Sherry et al., 2017). Abiotic biofilms developed in 96-well microplates, however, might differ from biotic epithelium-based biofilms in many aspects, including their resistance to antimicrobial agents (Bjarnsholt et al., 2013). We carried out antifungal susceptibility testing on Candida biofilms established in a mammalian vagina that closely mimic the clinical environment of human VVC. Though the mouse vagina differs from that of women in various physical aspects and may have different responses to Candida infections, it is still the preferred in vivo model for drug development, in the context of costeffectiveness and labor-intensity (Vecchiarelli et al., 2015).

Harriott et al. (2010) successfully demonstrated vaginal epithelium-based Candida biofilms using a mouse VVC model and raised the hypothesis that biofilm formation by Candida might be an initiating event for VVC (Harriott et al., 2010). The role of biofilms in the pathogenesis of VVC/RVVC was recently challenged by Swidsinski et al. (2019). Swidsinski et al. (2019) used fluorescent in situ hybridization (FISH) on vaginal biopsies from VVC patients and found no evidence of typical biofilm structures on vaginal epithelia or underlying tissues. It should be noted that biotic biofilms found in vivo often fail to present the typical three-dimensional mushroom structure, a biofilm morphology frequently reported by in vitro studies. In vivo biofilms uniquely grow as monolayers or microcolonies (Bjarnsholt et al., 2013), and detections of such structures often require experimental methods of higher resolutions such as SEM. From microbiological and clinical aspects, it is also reasonable to assume the involvement of Candida biofilms and persister cells in VVC/RVVC, as the recurrence pattern of RVVC coincides with the model of recurrent biofilm infections and persister cells proposed by Lewis (2010). Clinical isolates of C. albicans from the same RVVC patients over different periods of time, despite therapy, were found to be genetically identical, supporting the possible involvement of persister cells in the recurrence of RVVC (Fodor et al., 2002).

One of the major limitations of this study is that no other Candida species were included. Non-C. albicans Candida species have recently emerged as important etiological agents of VVC/RVVC (Richter et al., 2005; Gamarra et al., 2014). In contrast to $C$. albicans, some non-C. albicans Candida species are unable to form typical biofilms in vitro or in vivo, or are intrinsically resistant to many conventional antifungals (Guzel et al., 2013). A future study is required to examine the effect of polymers against VVC/RVVC caused by other Candida species.

\section{CONCLUSION}

This study systematically assessed the clinical potential of RAFT-derived polymethacrylates as an effective treatment for VVC/RVVC in a mouse model. Polymethacrylates effectively killed vaginal epithelium-related $C$. albicans in vivo by specially targeting biotic biofilms and persister cells, outperforming other market leading antifungals. Treatment presented negligible local toxicity.

\section{DATA AVAILABILITY STATEMENT}

The datasets generated for this study are available on request to the corresponding author.

\section{ETHICS STATEMENT}

The Ethics Review Boards of Wenzhou Medical University approved this study (wydw2016-0214). All animal experiments were carried out in accordance with the National Institutes of Health guide for the care and use of Laboratory animals.

\section{AUTHOR CONTRIBUTIONS}

YQ and XW conceived and designed the study. SZ, $\mathrm{XX}, \mathrm{LS}, \mathrm{BX}, \mathrm{WQ}, \mathrm{WZ}, \mathrm{KL}$, and YQ carried out the experiments. YQ, SZ, XW, and MD performed the data 
analysis. YQ wrote the manuscript. YQ, MD, and XW edited the manuscript. All authors reviewed the manuscript and provided critical comments.

\section{FUNDING}

This work was supported by the National Natural Science Foundation of China (Grant Number 81772241 to YQ); the National Natural Science Foundation of China (Grant Number 81873822 to XW); the 551 Talent Program of Wenzhou (to XW); and the Shenzhen Science and Technology Innovation Project (Grant Number JCY20170818100355168 to XW).

\section{REFERENCES}

Adjapong, G., Hale, M., and Garrill, A. (2017). A comparative investigation of azole susceptibility in Candida isolates from vulvovaginal candidiasis and recurrent vulvovaginal candidiasis patients in Ghana. Med. Mycol. 55, 686-689. doi: 10. 1093/mmy/myw122

Bjarnsholt, T., Alhede, M., Alhede, M., Eickhardt-Sorensen, S. R., Moser, C., Kuhl, M., et al. (2013). The in vivo biofilm. Trends Microbiol. 21, 466-474. doi: 10. 1016/j.tim.2013.06.002

Boikov, D. A., Locke, J. B., James, K. D., Bartizal, K., and Sobel, J. D. (2017). In vitro activity of the novel echinocandin CD101 at pH 7 and 4 against Candida spp. isolates from patients with vulvovaginal candidiasis. J. Antimicrob. Chemother. 72, 1355-1358. doi: 10.1093/jac/dkx008

Catalone, B. J., Kish-Catalone, T. M., Budgeon, L. R., Neely, E. B., Ferguson, M., Krebs, F. C., et al. (2004). Mouse model of cervicovaginal toxicity and inflammation for preclinical evaluation of topical vaginal microbicides. Antimicrob. Agents Chemother. 48, 1837-1847. doi: 10.1128/aac.48.5.18371847.2004

Chaillot, J., Tebbji, F., Garcia, C., Wurtele, H., Pelletier, R., and Sellam, A. (2017). $\mathrm{pH}$-dependant antifungal activity of valproic acid against the human fungal pathogen Candida albicans. Front. Microbiol. 8:1956. doi: 10.3389/fmicb.2017. 01956

Choukri, F., Benderdouche, M., and Sednaoui, P. (2014). In vitro susceptibility profile of 200 recent clinical isolates of Candida spp. to topical antifungal treatments of vulvovaginal candidiasis, the imidazoles and nystatin agents. J. Mycol. Med. 24, 303-307. doi: 10.1016/j.mycmed.2014.05.001

Crouss, T., Sobel, J. D., Smith, K., and Nyirjesy, P. (2018). Long-term outcomes of women with recurrent vulvovaginal candidiasis after a course of maintenance antifungal therapy. J. Low Genit. Tract Dis. 22, 382-386. doi: 10.1097/LGT. 0000000000000413

Dennerstein, G. J. (2017). Recurrent vulvovaginal candidiasis: a review of guidelines and recommendations. Aust. N. Z. J. Obstet. Gynaecol. 57, E12-E13.

Esposito, E., Campolo, M., Casili, G., Lanza, M., Filippone, A., Peritore, A. F., et al. (2018). Effect of pea protein plus grape seed dry extract on a murine model of Candida albicans induced vaginitis. Future Microbiol. 13, 1375-1382. doi: 10.2217/fmb-2018-0102

Fan, S., Liu, X., and Liang, Y. (2015a). Miconazole nitrate vaginal suppository 1,200 $\mathrm{mg}$ versus oral fluconazole $150 \mathrm{mg}$ in treating severe vulvovaginal candidiasis. Gynecol. Obstet. Invest. 80, 113-118. doi: 10.1159/000371759

Fan, S., Liu, X., Wu, C., Xu, L., and Li, J. (2015b). Vaginal nystatin versus oral fluconazole for the treatment for recurrent vulvovaginal candidiasis. Mycopathologia 179, 95-101. doi: 10.1007/s11046-014-9827-4

Fernandes Costa, A., Evangelista Araujo, D., Santos Cabral, M., Teles Brito, I., Borges De Menezes Leite, L., Pereira, M., et al. (2019). Development, characterization, and in vitro-in vivo evaluation of polymeric nanoparticles containing miconazole and farnesol for treatment of vulvovaginal candidiasis. Med. Mycol. 57, 52-62. doi: 10.1093/mmy/myx155

Fodor, E., Dosa, E., Nagy, A., Nagy, E., and Ferenczy, L. (2002). Karyotyping of Candida albicans and Candida glabrata isolates from recurrent vaginal

\section{ACKNOWLEDGMENTS}

KL wishes to acknowledge the CSIRO Julius Career Development Award for funding. The authors also would like to thank Margaret Smith and her team at Syndet Works, Australia for their assistance with preparing polymer gel formulations.

\section{SUPPLEMENTARY MATERIAL}

The Supplementary Material for this article can be found online at: https://www.frontiersin.org/articles/10.3389/fmicb. 2019.02592/full\#supplementary-material

infections by pulsed-field gel electrophoresis. Acta Microbiol. Immunol. Hung. 49, 59-68. doi: 10.1556/amicr.49.2002.1.6

Gamarra, S., Morano, S., Dudiuk, C., Mancilla, E., Nardin, M. E., De Los Angeles Mendez, E., et al. (2014). Epidemiology and antifungal susceptibilities of yeasts causing vulvovaginitis in a teaching hospital. Mycopathologia 178, 251-258. doi: $10.1007 /$ s1 1046-014-9780-2

Gao, M., Wang, H., and Zhu, L. (2016). Quercetin assists fluconazole to inhibit biofilm formations of fluconazole-resistant Candida albicans in in vitro and in vivo antifungal managements of vulvovaginal candidiasis. Cell Physiol. Biochem. 40, 727-742. doi: 10.1159/000453134

Grinceviciene, S., Bellen, G., Ruban, K., and Donders, G. (2017). Non-response to fluconazole maintenance treatment (ReCiDiF regimen) for recurrent vulvovaginal candidosis is not related to impaired glucose metabolism. Mycoses 60, 546-551. doi: 10.1111/myc. 12626

Guzel, A. B., Aydin, M., Meral, M., Kalkanci, A., and Ilkit, M. (2013). Clinical characteristics of Turkish women with Candida krusei vaginitis and antifungal susceptibility of the C. krusei isolates. Infect. Dis. Obstet. Gynecol. 2013:698736. doi: 10.1155/2013/698736

Harriott, M. M., Lilly, E. A., Rodriguez, T. E., Fidel, P. L. Jr., and Noverr, M. C. (2010). Candida albicans forms biofilms on the vaginal mucosa. Microbiology 156, 3635-3644. doi: 10.1099/mic.0.039354-0

Howley, M. M., Carter, T. C., Browne, M. L., Romitti, P. A., Cunniff, C. M., and Druschel, C. M. (2016). Fluconazole use and birth defects in the national birth defects prevention study. Am. J. Obstet. Gynecol. 214, e651-e659. doi: 10.1016/j.ajog.2015.11.022

Ilkit, M., and Guzel, A. B. (2011). The epidemiology, pathogenesis, and diagnosis of vulvovaginal candidosis: a mycological perspective. Crit. Rev. Microbiol. 37, 250-261. doi: 10.3109/1040841X.2011.576332

Kasper, L., Miramon, P., Jablonowski, N., Wisgott, S., Wilson, D., Brunke, S., et al. (2015). Antifungal activity of clotrimazole against Candida albicans depends on carbon sources, growth phase and morphology. J. Med. Microbiol. 64, 714-723. doi: 10.1099/jmm.0.000082

Lafleur, M. D., Kumamoto, C. A., and Lewis, K. (2006). Candida albicans biofilms produce antifungal-tolerant persister cells. Antimicrob. Agents Chemother. 50, 3839-3846. doi: 10.1128/aac.00684-06

Lafleur, M. D., Qi, Q., and Lewis, K. (2010). Patients with long-term oral carriage harbor high-persister mutants of Candida albicans. Antimicrob. Agents Chemother. 54, 39-44. doi: 10.1128/AAC.00860-09

Lewis, K. (2010). Persister cells. Annu. Rev. Microbiol. 64, 357-372. doi: 10.1146/ annurev.micro.112408.134306

Locock, K. E., Meagher, L., and Haeussler, M. (2014). Oligomeric cationic polymethacrylates: a comparison of methods for determining molecular weight. Anal. Chem. 86, 2131-2137. doi: 10.1021/ac403735n

Mendling, W., Brasch, J., Cornely, O. A., Effendy, I., Friese, K., GinterHanselmayer, G., et al. (2015). Guideline: vulvovaginal candidosis (AWMF 015/072), S2k (excluding chronic mucocutaneous candidosis). Mycoses 58(Suppl. 1), 1-15. doi: 10.1111/myc.12292

Mikamo, H., Matsumizu, M., Nakazuru, Y., Okayama, A., and Nagashima, M. (2015). Efficacy and safety of a single oral $150 \mathrm{mg}$ dose of fluconazole for 
the treatment of vulvovaginal candidiasis in Japan. J. Infect. Chemother. 21, 520-526. doi: 10.1016/j.jiac.2015.03.011

Molgaard-Nielsen, D., Svanstrom, H., Melbye, M., Hviid, A., and Pasternak, B. (2016). Association between use of oral fluconazole during pregnancy and risk of spontaneous abortion and stillbirth. JAMA 315, 58-67.

Muzny, C. A., and Schwebke, J. R. (2015). Biofilms: an underappreciated mechanism of treatment failure and recurrence in vaginal infections. Clin. Infect. Dis. 61, 601-606. doi: 10.1093/cid/civ353

Netea, M. G., Simon, A., Van De Veerdonk, F., Kullberg, B. J., Van Der Meer, J. W., and Joosten, L. A. (2010). IL-1beta processing in host defense: beyond the inflammasomes. PLoS Pathog. 6:e1000661. doi: 10.1371/journal.ppat.100 0661

Qu, Y., Locock, K., Verma-Gaur, J., Hay, I. D., Meagher, L., and Traven, A. (2016). Searching for new strategies against polymicrobial biofilm infections: guanylated polymethacrylates kill mixed fungal/bacterial biofilms. J. Antimicrob. Chemother. 71, 413-421. doi: 10.1093/jac/dkv334

Richter, S. S., Galask, R. P., Messer, S. A., Hollis, R. J., Diekema, D. J., and Pfaller, M. A. (2005). Antifungal susceptibilities of Candida species causing vulvovaginitis and epidemiology of recurrent cases. J. Clin. Microbiol. 43, 2155-2162. doi: $10.1128 / \mathrm{jcm} .43 .5 .2155-2162.2005$

Sherry, L., Kean, R., Mckloud, E., O'donnell, L. E., Metcalfe, R., Jones, B. L., et al. (2017). Biofilms formed by isolates from recurrent vulvovaginal candidiasis patients are heterogeneous and insensitive to fluconazole. Antimicrob. Agents Chemother. 61:e1065-17. doi: 10.1128/AAC.01065-17

Sobel, J. D. (2016). Recurrent vulvovaginal candidiasis. Am. J. Obstet. Gynecol. 214, 15-21. doi: 10.1016/j.ajog.2015.06.067

Swidsinski, A., Guschin, A., Tang, Q., Dorffel, Y., Verstraelen, H., Tertychnyy, A., et al. (2019). Vulvovaginal candidiasis: histologic lesions are primarily polymicrobial and invasive and do not contain biofilms. Am. J. Obstet. Gynecol. 220:e91. doi: 10.1016/j.ajog.2018.10.023

Tapia, C. V., Hermosilla, G., Fortes, P., Alburquenque, C., Bucarey, S., Salinas, H., et al. (2017). Genotyping and persistence of Candida albicans from pregnant women with vulvovaginal candidiasis. Mycopathologia 182, 339-347. doi: 10. 1007/s11046-016-0095-3

Vecchiarelli, A., Gabrielli, E., and Pericolini, E. (2015). Experimental models of vaginal candidiasis and inflammation. Future Microbiol. 10, 1265-1268. doi: 10.2217/fmb. 15.52

Vogl, T., Eisenblatter, M., Voller, T., Zenker, S., Hermann, S., Van Lent, P., et al. (2014). Alarmin S100A8/S100A9 as a biomarker for molecular imaging of local inflammatory activity. Nat. Commun. 5:4593. doi: 10.1038/ncomms5593

Wächtler, B., Wilson, D., and Hube, B. (2011). Candida albicans adhesion to and invasion and damage of vaginal epithelial cells: stage-specific inhibition by clotrimazole and bifonazole. Antimicrob. Agents Chemother. 55, 4436-4439. doi: 10.1128/AAC.00144-11

Yang, S., Hay, I. D., Cameron, D. R., Speir, M., Cui, B., Su, F., et al. (2015). Antibiotic regimen based on population analysis of residing persister cells eradicates Staphylococcus epidermidis biofilms. Sci. Rep. 5:18578. doi: 10.1038/srep18578

Zhang, S. S., Xia, W. T., Xu, J., Xu, H. L., Lu, C. T., Zhao, Y. Z., et al. (2017). Three-dimensional structure micelles of heparin-poloxamer improve the therapeutic effect of 17beta-estradiol on endometrial regeneration for intrauterine adhesions in a rat model. Int. J. Nanomed. 12, 5643-5657. doi: 10.2147/IJN.S137237

Conflict of Interest: The authors declare that the research was conducted in the absence of any commercial or financial relationships that could be construed as a potential conflict of interest.

Copyright (c) $2019 \mathrm{Wu}$, Zhang, Xu, Shen, Xu, Qu, Zhuang, Locock, Deighton and Qu. This is an open-access article distributed under the terms of the Creative Commons Attribution License (CC BY). The use, distribution or reproduction in other forums is permitted, provided the original author(s) and the copyright owner(s) are credited and that the original publication in this journal is cited, in accordance with accepted academic practice. No use, distribution or reproduction is permitted which does not comply with these terms. 